\title{
Study of Influence of Electrode Geometry on Impedance Spectroscopy
}

\author{
Riaz Ahmed ${ }^{1, *}$ Ken Reifsnider ${ }^{2}$ \\ ${ }^{1}$ Dept. of Mechanical Engineering, Khulna University of Engineering \& Technology, Khulna - \\ 920300, Bangladesh, \\ ${ }^{2}$ University of South Carolina, Columbia, SC 29208, USA \\ *E-mail: riaz.usc@gmail.com
}

Received: 28 November 2010 / Accepted: 18 December 2010 / Published: 1 April 2011

\begin{abstract}
Electrochemical Impedance Spectroscopy (EIS) is a powerful and proven tool for analyzing AC impedance response. A conventional three electrode EIS method was used to perform the investigation in the present study. Saturated potassium chloride solution was used as the electrolyte and three different material rods were used as working electrodes. Different configurations of electrode area were exposed to the electrolyte as an active area to investigate electrode geometry effects. Counter to working electrode distance was also altered while keeping the working electrode effective area constant to explore the AC response dependence on the variation of ion travel distance. Some controlled experiments were done to validate the experimental setup and to provide a control condition for comparison with experimental results. A frequency range of $100 \mathrm{mHz}$ to $1 \mathrm{MHz}$ was used for all experiments. In our analysis, we have found a noteworthy influence of electrode geometry on AC impedance response. For all electrodes, impedance decreases with the increase of effective area of the electrolyte. High frequency impedance is not as dependent on geometry as low frequency response. The observed phase shift angle drops in the high frequency region with increased working electrode area, whereas at low frequency the reverse is true. Resistance and capacitive reactance both decrease with an increase of area, but resistance response is more pronounce than reactance. For lower frequencies, small changes in working area produce very distinctive EIS variations. Electrode material as well as geometry was systematically varied in the present study. From these and other studies, we hope to develop a fundamental foundation for understanding specific changes in local geometry in fuel cell (and other) electrodes as a method of designing local morphology for specific performance.
\end{abstract}

Keywords: SOFC, electrode, geometry, EIS

\section{INTRODUCTION}

Solid oxide fuel cells (SOFCs) are considered as one of the most promising systems for energy conversion in near future due to their very high electrical efficiency and the possibility to operate with 
lower or zero emissions.[1,2] Current SOFC technology typically uses porous materials that have little or no ionic conduction for the electrodes. In particular, porous $\mathrm{LaMnO}_{3}$ is commonly used as the cathode material. Because $\mathrm{LaMnO}_{3}$ is largely an electronic-only conductor, high porosity is critical for increasing the number of active regions for oxygen reduction in the electrochemical conversion. The geometry and dimensions of SOFCs can affect the performance of the fuel cell. However, effects due to changes to the design of the SOFCs can be complicated, requiring trial and error to improve the fuel cell. In particular, the specific effects of changing the thickness of the cathode for fuel cell performance can be difficult to determine[3].

Solid oxide fuel cell (SOFC) systems for aircraft applications require an order of magnitude increase in specific power density $(1.0 \mathrm{kWkg}-1)$ and long life. While significant research is underway to develop anode supported cells which operate at temperatures in the range of $650-800{ }^{\circ} \mathrm{C}$, concerns about $\mathrm{Cr}$-contamination from the metal interconnect may drive the operating temperature down further, to $750{ }^{\circ} \mathrm{C}$ and lower. Higher temperatures, $850-1000{ }^{\circ} \mathrm{C}$, are more favorable in order to achieve specific power densities of $1.0 \mathrm{kWkg}-1$. Since metal interconnects are not practical at these high temperatures and can account for up to $75 \%$ of the weight of the stack, NASA is pursuing a design that uses a thin, LaCrO3- based ceramic interconnect that incorporates gas channels into the electrodes. The bi-electrode supported cell (BSC) uses porous YSZ scaffolds, on either side of a 10-20 $\mu \mathrm{m}$ electrolyte. The key feature of the design concept is the symmetrical cell, which is made by supporting the thin electrolyte on both sides with a porous YSZ support structure, thus the name bi-supported cell or BSC[4]. In our present study, we are focusing on BSC concept and figure 1 showing such electrolyte supported structure.

Generally, the cathode and anode of a fuel cell are made of porous materials. Figure 2 shows the porous anode and cathode of a BSC structure. Porous materials play two key roles in fuel cell technology. The first is that of transporting gases to/from the fuel cell electrodes. [5].
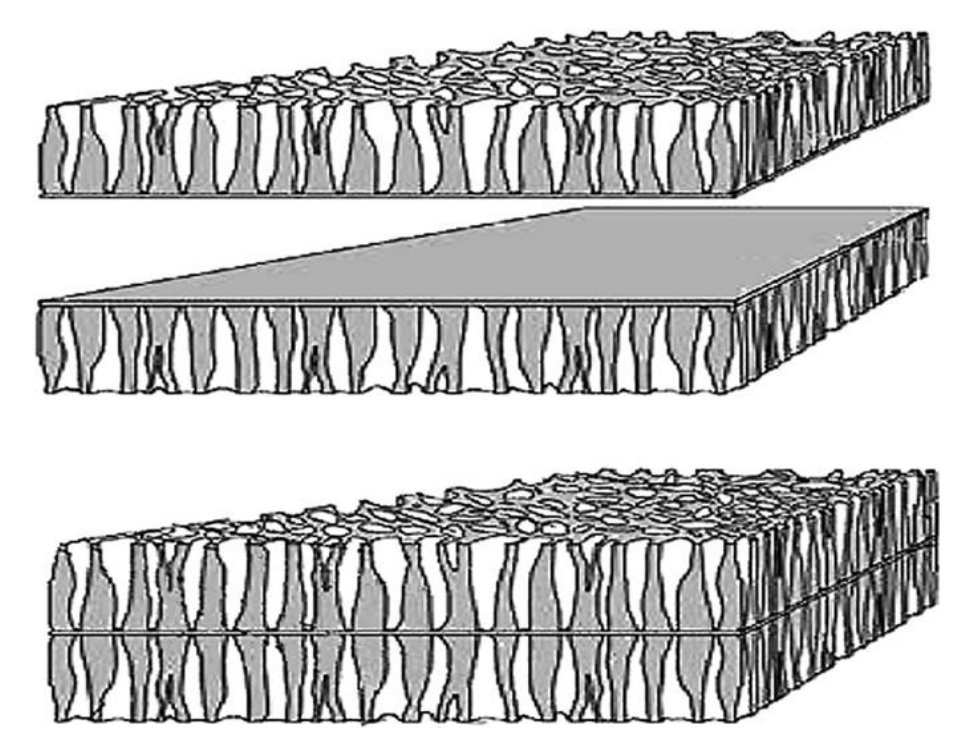

Figure 1. Thin YSZ electrolyte supported between two graded porous scaffolds[4]. 


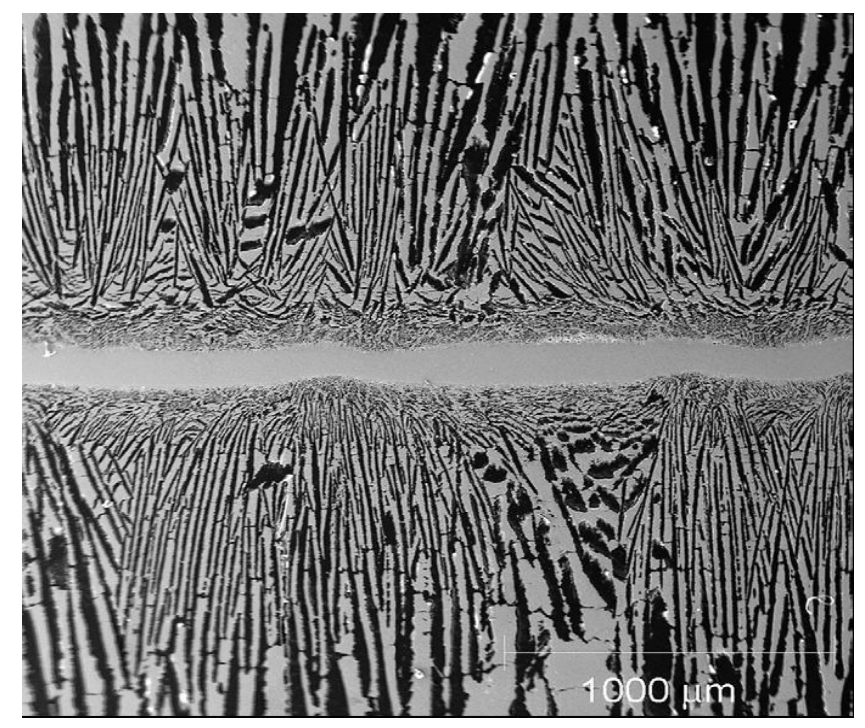

Figure 2. Porous anode and cathode of a BSC structure[4].

The second vital role of porous materials is their electrochemical function as an anode or cathode. In both PEMFCs and SOFCs, the electrodes play a vital role in minimizing losses attributable to electrode kinetics, and in some cases mass transport. This is achieved by maximizing the length of the so-called triple phase or three-phase boundary (TPB), a term describing the conjunction of a pore space, an ionically conducting phase, and an electronically conducting phase. In practice this is achieved by the use of porous composite electrode structures containing both ionically and electronically conducting materials[5].

The importance of local morphology has been recognized in recent years because of its large influence on fuel cell performance. Numerous papers have been published that discuss local geometry effects on half-cell performance by different Investigators. However, of the papers that do investigate the effect of cell geometry on the reliability of the determination of electrochemical parameters[6,7], only some[8-10] provide experimental data to validate the conclusions reached by mathematical modeling. A change of morphology of the cathode with time (decreasing three phase contact area and limiting gas diffusion) and interfacial reactions between the cathode and electrolyte during operation can limit the life of SOFCs and must be minimized[11]. However, these and other discussions address morphology only in terms of bulk properties, such as particle size, particle neck size, tortuosity, porosity, and total triple phase boundary length.[12,13] Modern nano-manufacturing methods have given us the option of designing the specific local geometry for optimum performance, but the fundamental understanding, knowledge, and analytical models to specify such local details in multifunctional materials is not in the literature.

In our present work, we selected the simplest possible control experiment to advance this understanding, the simple electrochemical cell, for which there is abundant literature to describe the general behavior.[10,14] What is remarkable is that discussions of the effect of specific local geometry on electrochemical behavior are sparse. In the present case, our primary interest was to gain greater understanding of the effect of local morphology on impedance response, as a foundation for developing impedance spectroscopy (EIS) as a global method of assessing and evaluating local 
morphology. To isolate those details, we tried to observe changes in impedance response caused by altering the active area and geometry of the cathode.

In this study, we were only concentrated on changing only one geometry parameter at a time that can influence the cell performance (such as Cathode Active Area, Ion Traveling Distance etc.). To eliminate other parameters (Chemical Reaction, Poisoning, Current Density etc.) that may influence cell performance, a very high conductive aqueous solution was used as the electrolyte. We also conducted a set of cyclic voltammetry tests to confirm that the oxidation/chemical reaction was negligible in our EIS experiments.

\section{EXPERIMENTAL TECHNIQUE}

A conventional three-electrode method was used for experimental investigations. Three different materials (copper, brass and aluminum) were used as working electrodes. Copper and brass are high-conductivity electrical conductors compared to Aluminum.

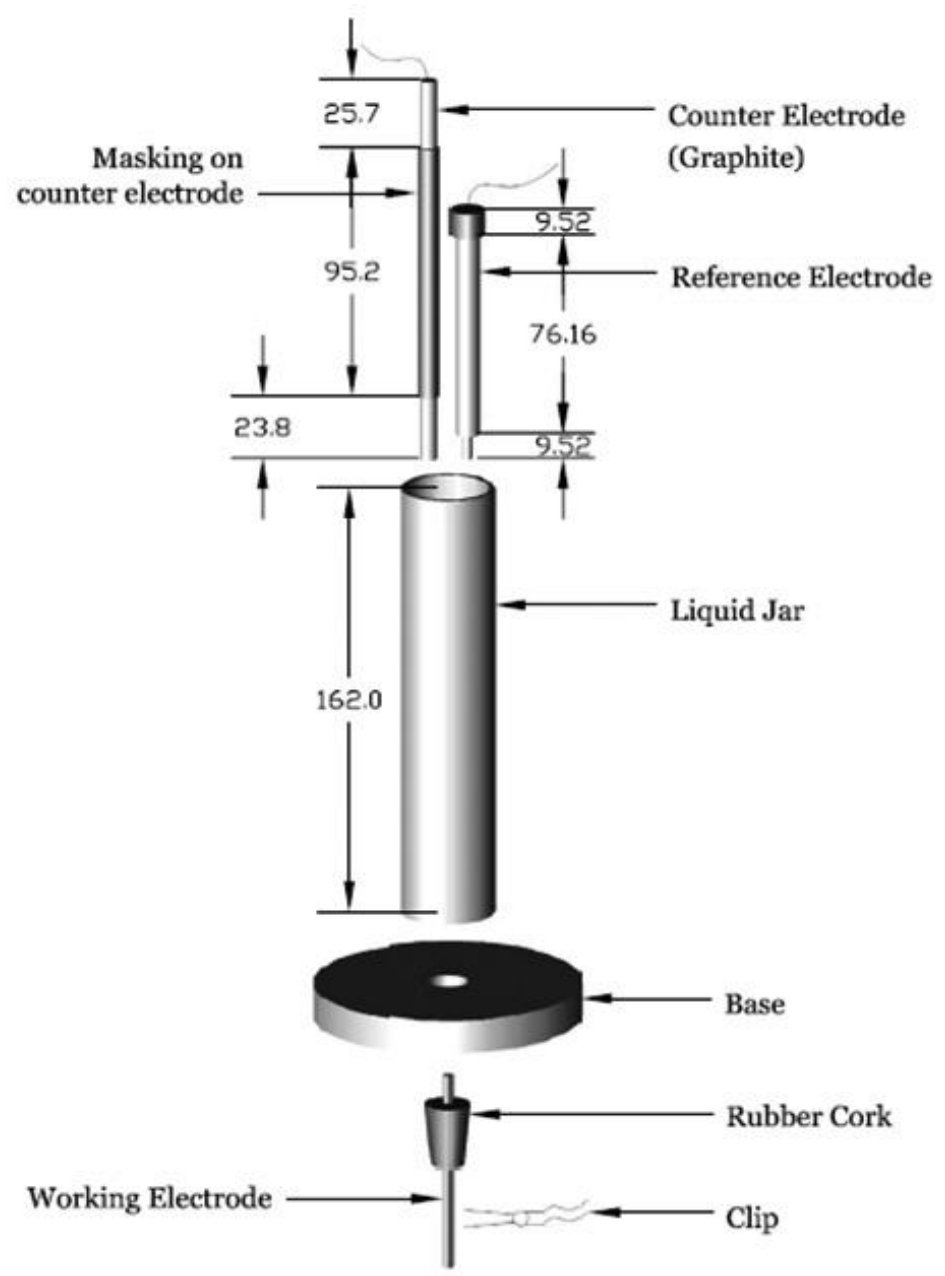

Figure 3. Experimental setup (all measurements in $\mathrm{mm}$ ) 
Electrode materials were rod shaped and $3 \mathrm{~mm}$ in diameter. Graphite rod was used as counter electrodes and saturated $\mathrm{KCl}$ solution served as the electrolyte. A Luggin capillary with saturated $\mathrm{KCl}$ solution was used as a reference electrode. For some experiments, to validate experimental setup and for establishing some controlled results, alumina was used as a working electrode with $\mathrm{KCl}$ solution as the electrolyte and in other setup, Deionized (DI) water was used as the electrolyte with brass/copper as the working electrode. A Faraday cage was used throughout the experimental sessions to isolate the outcomes from electromagnetic radiation and noise. An REF600 potentiostat was used from Gamry Instrument Corp. for both EIS and Cyclic Voltammetry tests.

Before using the electrode for experiments, the surface was decontaminated by Muriatic Acid and Distilled Water. A portion of the surface of the counter electrode was sealed by a rubber tube in its middle to insure that in every experiment the counter electrode active area protruding into the electrolyte remained unchanged. A small rubber cap was also used on one end (exposed side on electrolyte) of the working electrode to avoid any local singularity effect.

Approximately $120 \mathrm{~mm}$ electrodes were used for the experiments. One end of the electrode was exposed to the electrolyte and capped by the rubber cap, and other end was clamped with a caliper and connected to the Gamry potentiostat through an alligator clip.

The caliper was used to advance the electrode upward, which increased the active area of the working electrode exposed to the electrolyte. To maintain a constant ion travel distance for all experiments, when the working electrode exposed length was increased in the electrolyte, the counter electrode was also moved upward for the same length, except for the experiments where ion travel distance was altered deliberately while keeping working electrode area unchanged. Following every test, electrodes (working, counter and reference) and the electrolyte jar were cleaned by distilled water. A complete view of the experimental setup is shown in figure 3.

A frequency range of $100 \mathrm{mHz}$ to $1 \mathrm{MHz}$ was used for all tests. During the experiments, the frequency varied from an upper limit $(1 \mathrm{MHz})$ to the lower limit $(100 \mathrm{mHz})$. The AC potential was set at $10 \mathrm{mV}$ rms and a DC potential was measured against open circuit voltage (Eoc).

\section{RESULTS AND DISCUSSION}

\subsection{Control Results}

To validate the experimental setup, two controlled experiments were done. In the first experiment, an alumina rod (ceramic) was used as the working electrode and $\mathrm{KCl}$ solution as the electrolyte. This is a fully capacitive system wherein alumina acts as pure insulator. In other experiments, brass and copper rods were used as working electrodes with Deionized water (DI water) as the electrolyte.

\subsubsection{Alumina as working electrode}

Alumina is a ceramic and fully an insulator. When alumina was used as the working electrode, the system response was nearly identical to a parallel plate capacitor. With a decrease of frequency, 
impedance was observed to increases linearly, as the growth of ion density was linear in the electrolyte. Since alumina is an insulator, the active area exposed to the electrolyte solution did not cause any change in the impedance response, as shown in figure-4. Also, the impedance values were very high, as expected.

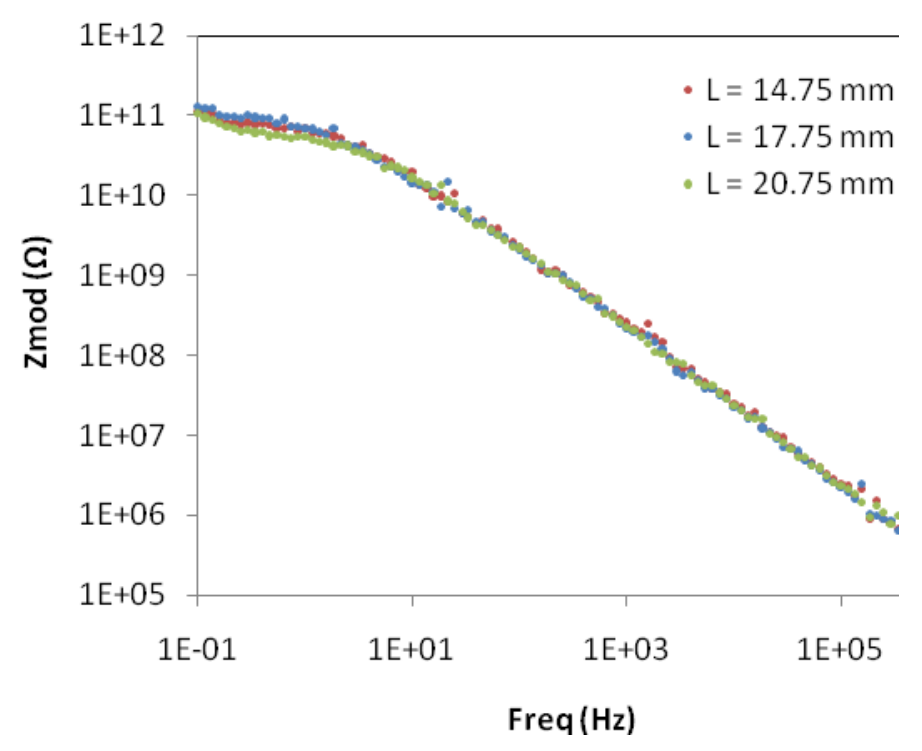

Figure 4. Impedance response for alumina working electrode for varied electrode exposure areas.

\subsubsection{DI water as electrolyte}

Deionization is the removal of ions - positive (cation) and negative (anion) - from the water.

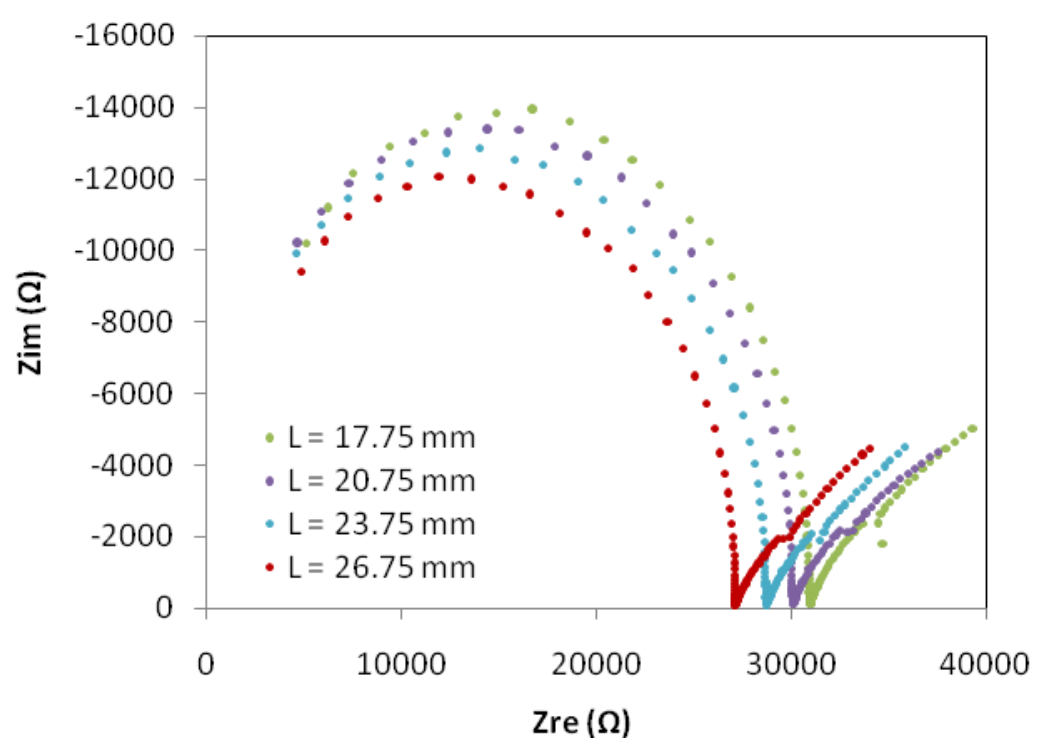

Figure 5. Nyquist plot for brass using DI water as electrolyte for varied active area. 


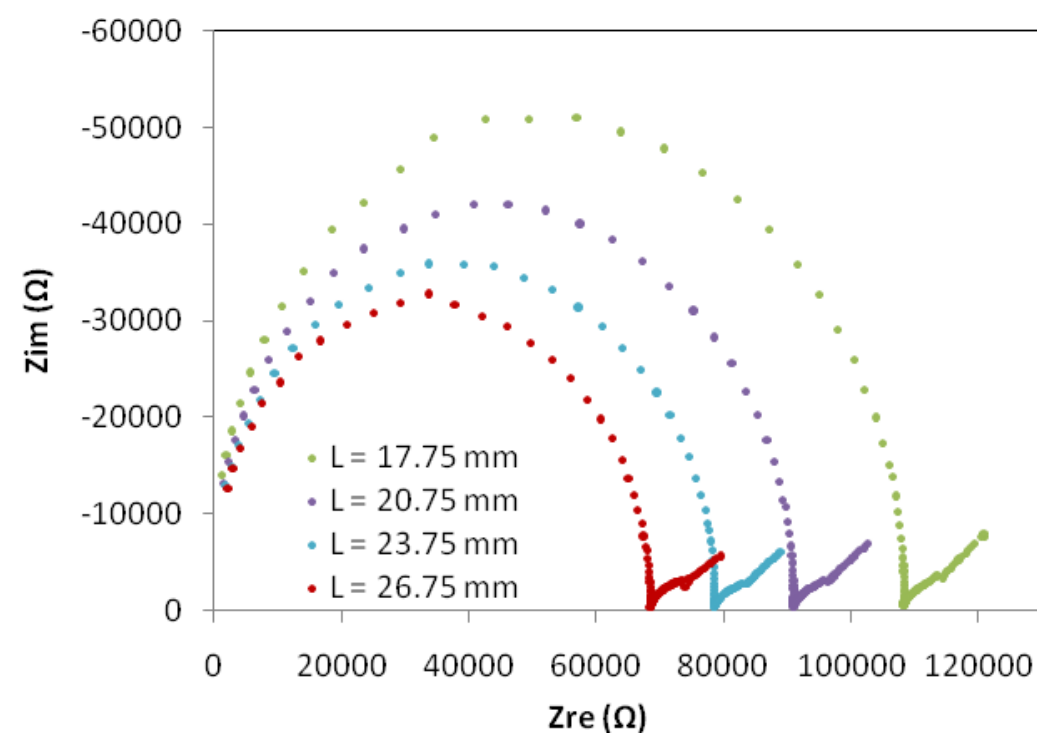

Figure 6. Nyquist plot for copper using DI water as electrolyte for varied active area.

The disadvantage of deionized water is that it is very corrosive to metal. Since DI water has no dissolved solids, water will seek equilibrium with whatever it contacts. It will react with anything it comes in contact with, especially metals and alloys like calcium, magnesium, copper, brass and stainless steel.

Four different active areas of electrode were used to observe changes in impedance response when DI water was used as the electrolyte. In Figures 5 and 6, semicircular arcs were observed from Nyquist plots, as well as $45^{\circ}$ straight lines to the Zreal axis. These semicircles represent charge transfer resistance while the $45^{\circ}$ lines represent Warburg impedance. Warburg Impedance is associated with diffusion. Diffusion starts at almost the same frequency for all electrode lengths (L) in the low frequency region. The DI water is an ion free liquid that attempts to bring the solution to equilibrium when it comes in contact with the metal electrode by collecting ions from the solid, after which diffusion starts. Due to the slowness of the process, diffusion occurs only in the low frequency region. We also observed that the active working electrode length alteration had a significant influence on the impedance response for this case. The influence of working electrode active area on impedance response is discussed briefly in following section.

\subsection{Response due to variation of working electrode active area}

Seven different electrode exposure lengths were chosen to vary the active area of the working electrode. A length of $14.75 \mathrm{~mm}$ was set as the initial exposed length of the working electrode into the electrolyte. Subsequently, the electrode exposure length into electrolyte was increased using the calipers, attached to other side of the electrode. Each increment of motion increased the exposed length by $3 \mathrm{~mm}$ into the electrolyte. As oxidation was very slight on the electrode surface (as established from voltammetry tests), only a single material was used to examine the response due to area alteration for each experiment, rather than using a different piece of material for each active area. 
The measured impedance response for different electrode materials with the variation of active electrode area is shown in Figures 8-10. The Bode plot is segmented into two parts to provide more clarity in observing the impedance response. Figures 8 and 10 illustrate that impedance as well as resistance (the real part of impedance) and capacitive reactance (the imaginary part of impedance) decreased with an increase of active surface area of the electrode in the electrolyte.

We anticipated that our experimental setup response would follow the equivalent Randles circuit (Figure 7):

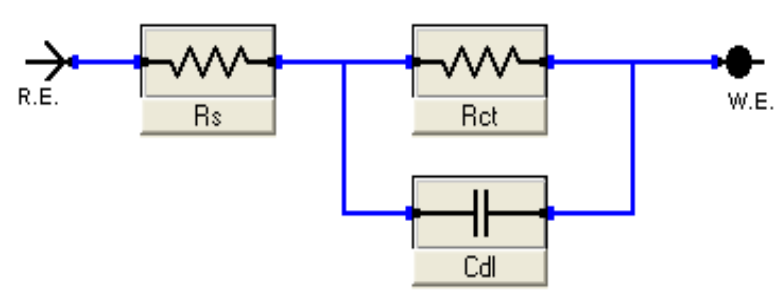

Figure 7. Simplified Randles Circuit

By solving the circuit,

$$
\begin{aligned}
& Z_{r e}=R_{s}+\frac{R_{c t}}{1+\omega^{2} R_{c t}{ }^{2} C_{d l}{ }^{2}} \\
& Z_{i m}=-\frac{\omega R_{c t}{ }^{2} C_{d l}}{1+\omega^{2} R_{c t}{ }^{2} C_{d l}{ }^{2}}
\end{aligned}
$$

Electrical Impedance,

$$
Z^{2}=Z_{r e}{ }^{2}+Z_{i m}{ }^{2}
$$

Again, Capacitance can be rewritten as:

$$
C_{d l}=\varepsilon_{r} \varepsilon_{0} \frac{A}{d}
$$

Equation (1) and (2) can be rearranged as:

$$
\begin{aligned}
& Z_{r e}=R_{s}+\frac{R_{c t} d^{2}}{d^{2}+\omega^{2} R_{c t}^{2}\left(\varepsilon_{r} \varepsilon_{0} A\right)^{2}} \\
& Z_{i m}=-\frac{\omega R_{c t}{ }^{2}\left(\varepsilon_{r} \varepsilon_{0} A\right) d}{d^{2}+\omega^{2} R_{c t}^{2}\left(\varepsilon_{r} \varepsilon_{0} A\right)^{2}}
\end{aligned}
$$


For our experiments, active area (A) can be calculated as:

$$
A=\pi D L
$$

With an increase of interfacial surface area (Active area) between working electrode and electrolyte, the electrode is allowing more regions to avoid collecting charge. At high frequency, the active electrode area is fresh enough to avoid surface charges. Thus, the active surface area does not play an appreciable role and electrolyte resistance $\left(R_{s}\right)$ is only responsible for impedance response. When the oscillation frequency decreases, a very thin film of charge starts growing on the electrodeelectrolyte interface with time. Owing to the formation of this film, charge transfer resistance increases with a decrease of frequency, which eventually increases impedance.

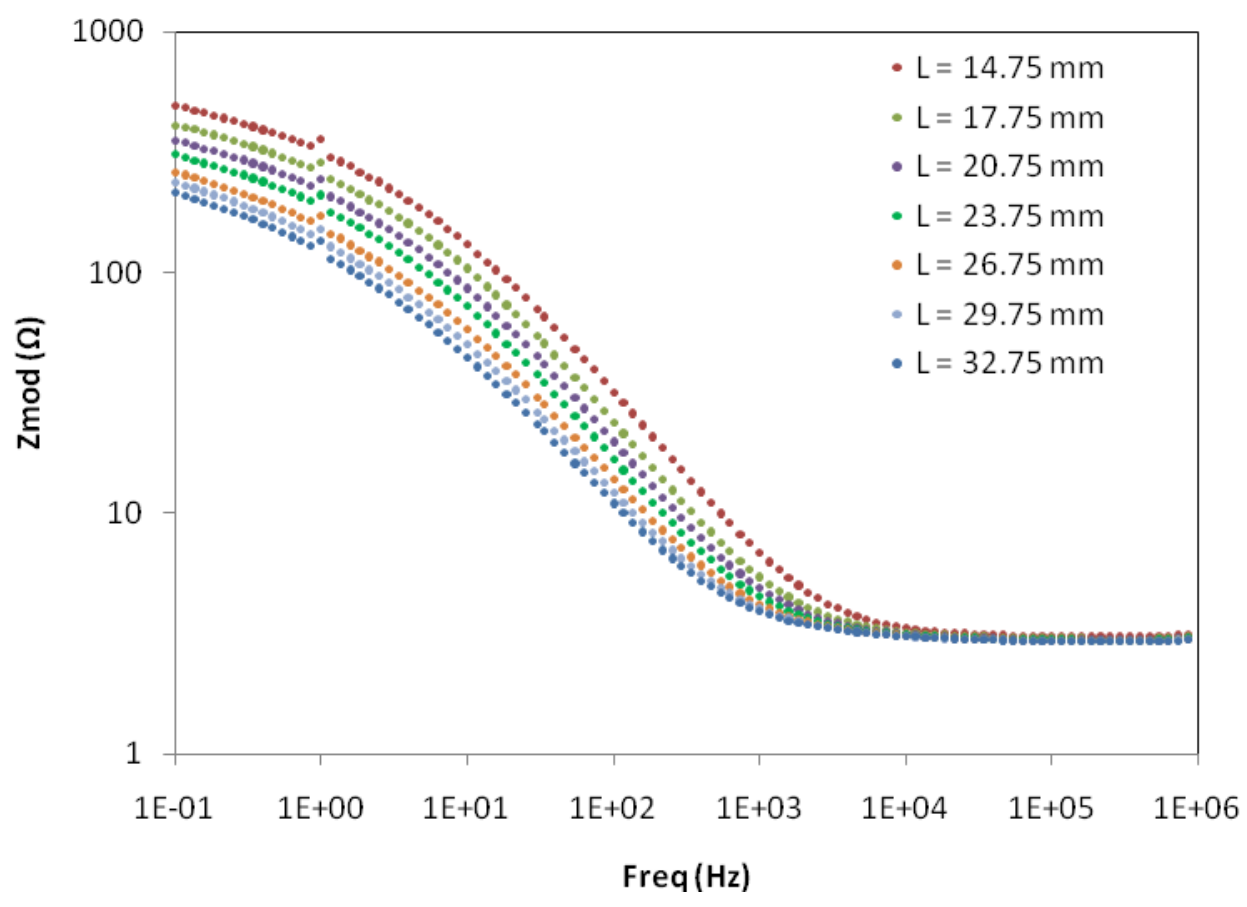

Figure 8. Magnitude of impedance response with frequency for different active area of Copper electrode

In the low frequency region, the effective surface area is a significant feature. Because large surface area allows more charge to pass by and thus reduces impedance. An initial impedance of approximately $3 \mathrm{ohm}$ was observed from the impedance plots. This impedance resulted the collective effect of the electrolyte and other system resistances. In all plots, there is a point observed at $1 \mathrm{~Hz}$, which is not continuous with the whole plot. We later determined that this extraneous point comes from ambient electromagnetic fields that penetrated the Faraday cage. 


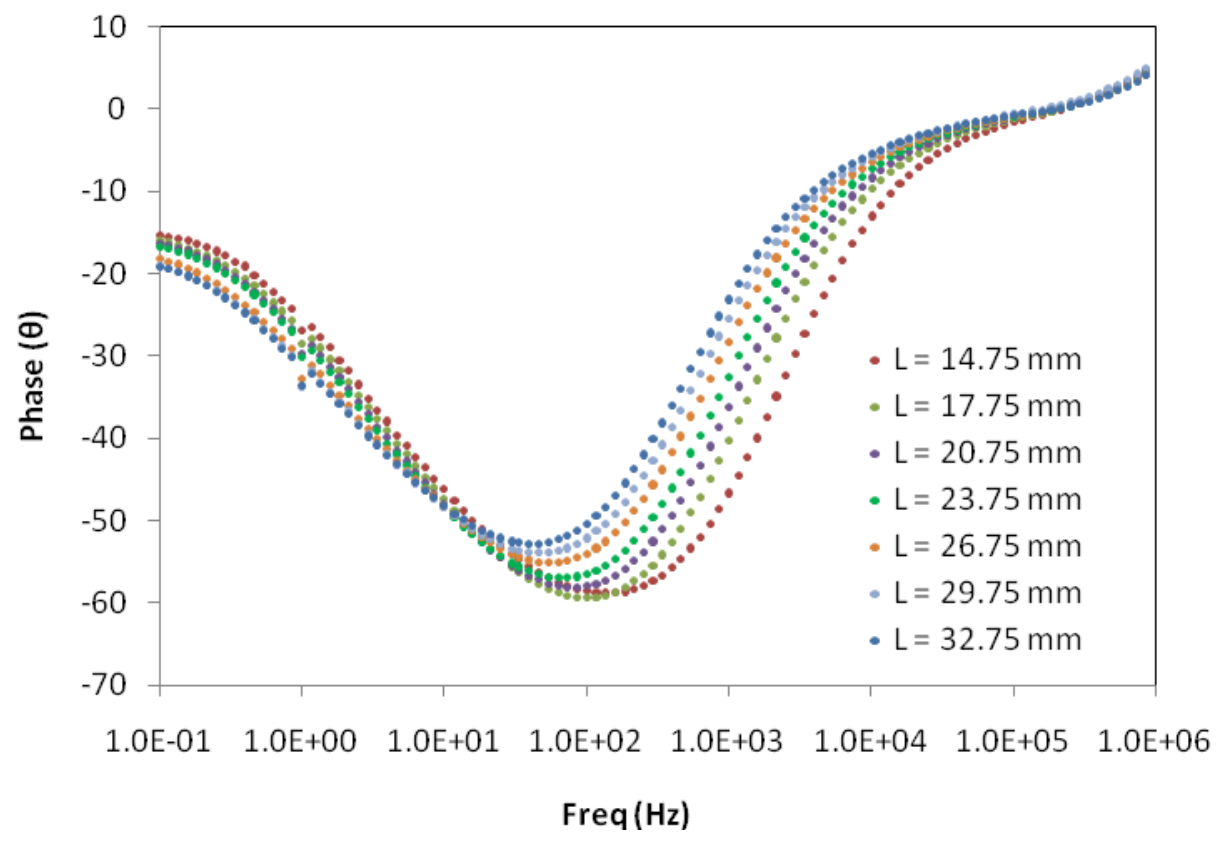

Figure 9. Phase shift variation with frequency for different active area of Copper electrode.

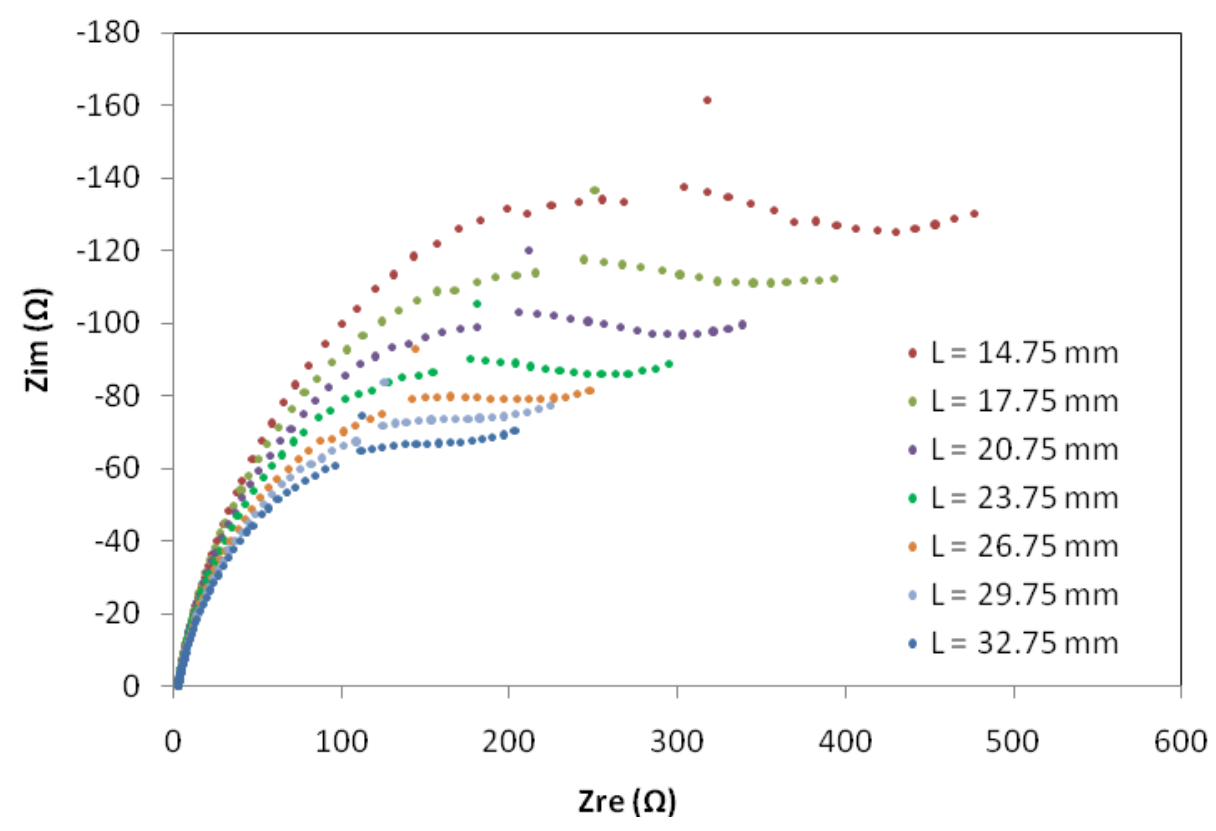

Figure 10. Nyquist plot for different active area of Copper electrode

Figure 9 shows the phase angle feedback for area alteration. For all materials, the high frequency region is independent of surface area. In the intermediate frequency region the phase angle decreases with an increase of active area and in the low frequency region it shows the opposite phenomenon. However, the intermediate frequency region is more sensitive than the low frequency 
region. In the intermediate frequency region, the resistance is more dominant then capacitive reactance, and in the low frequency region, it has the reverse behavior with an increase of active area. For both Copper and Brass, the minimum phase angle decreases with exposure area.

Figures 8-10 show the impedance response for the solid form of copper electrode. These experiments were also done for Brass and Aluminum electrodes as well. A hollow electrode structure of all materials was also used for the same setup and observed roughly the same phenomena for both forms of materials.

\subsection{Response due to change of ion travel distance}

Keeping all other parameters unchanged, only the counter electrode was moved vertically to vary the Ion travel distance $(\mathrm{M})$ for this series of experiments. Copper was used as the working electrode and the active electrode length was $14.75 \mathrm{~mm}$ for all tests. Initially, $64 \mathrm{~mm}$ was the electrode spacing. Subsequently, the counter electrode was moved upward a distance of $8 \mathrm{~mm}$ for each test.

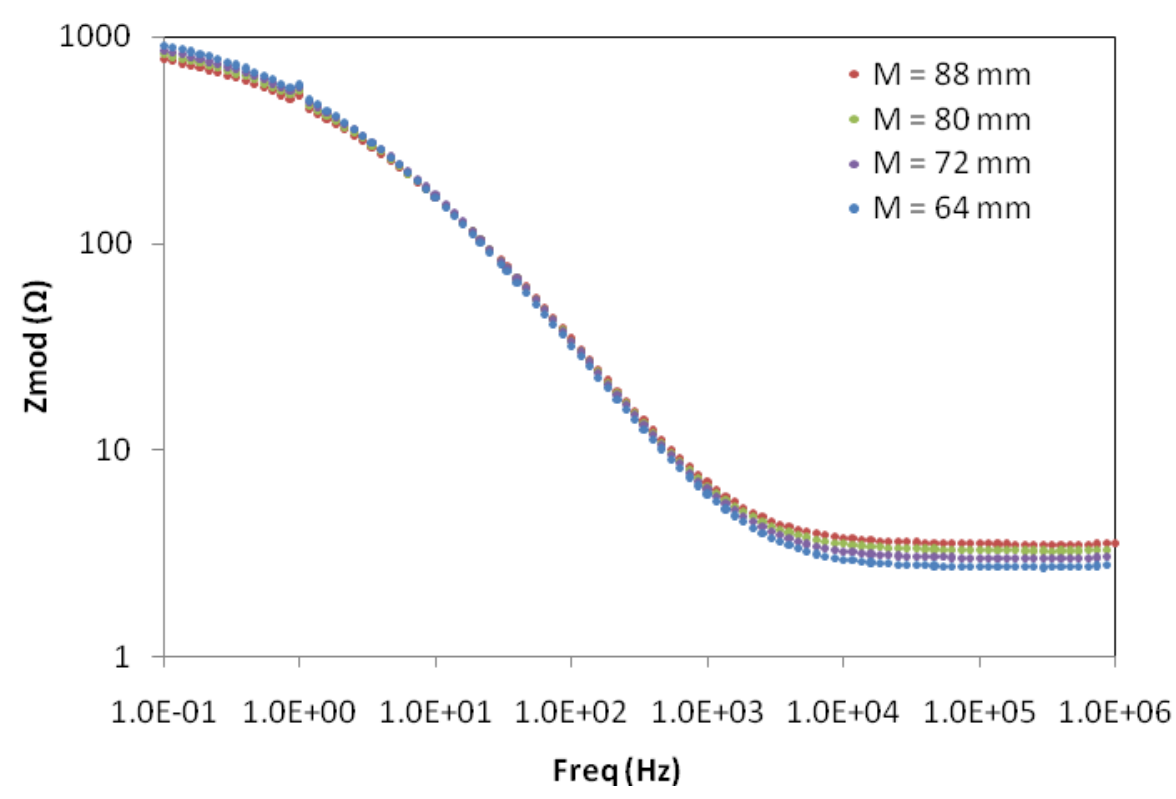

Figure 11. Impedance response for varied ion travel distance.

As we have seen earlier, in the high frequency region, the electrolyte resistance is the key factor controlling the impedance response. In the high frequency region, the electrolyte resistance increased with the increase of ion travelling distance (figure 11). However, the electrolyte resistance is reasonably low compared to total resistance. At low frequency, due to the growth of very thin film thickness on electrode-electrolyte interface, the local charge density is significant for total impedance response, since film thickness is dependent on charge density. In this area, charge transfer resistance is low for extended values of $\mathrm{M}$, because of lower charge density (figure 12). 


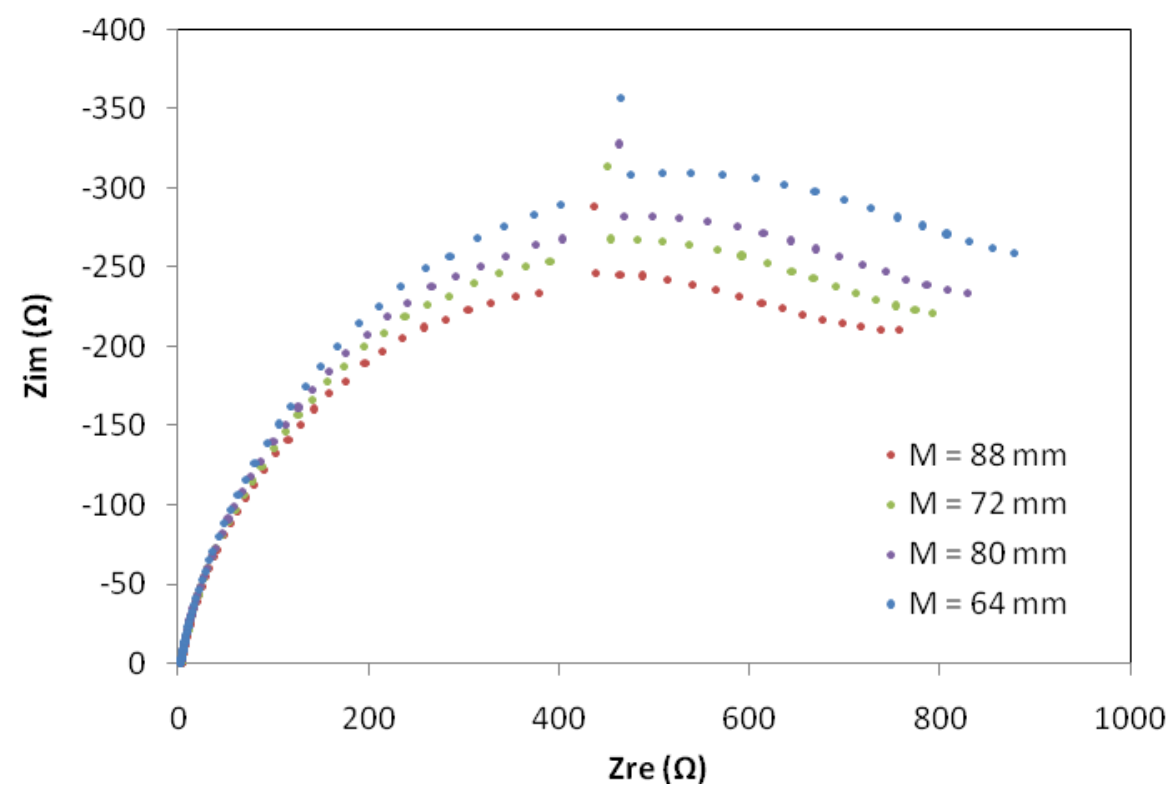

Figure 12. Nyquist plot for varied ion travel distance.

\subsection{Comparison with control results}

From section-3.2 it was observed that the measured impedance parameters vary with changes of active working electrode area, which is also true for section-3.1.2 (where DI water was the electrolyte), whereas active area variation doesn't affect the response for section-3.1.1 (alumina as a working electrode). As alumina has an absolute resistance to charge transfer, it is expected that it will show a linear variation of impedance with frequency, i.e., behave like a pure capacitor. Conversely, copper and brass are good conductors, so that active surface area is a controlling factor for those cases. At a very high frequency of excitation, due to very low charge transfer resistance, electrolyte resistance is the only active contributor and shows a flat impedance curve for good conductors, whereas for alumina, the impedance increases linearly from the very beginning because of its very high charge transfer resistance.

Table1. Parametric values calculated for DI water test

\begin{tabular}{|ccccc|}
\hline Length & \multicolumn{2}{c}{ Copper } & \multicolumn{2}{c|}{ Brass } \\
$(\mathrm{mm})$ & $R_{c t}(\mathrm{ohm})$ & $C_{d l}$ & $R_{c t}(\mathrm{ohm})$ & $C_{d l}$ \\
\hline 17.75 & 103432.63 & $1.28 \mathrm{E}-11$ & 25927.79 & $2.30 \mathrm{E}-11$ \\
\hline 20.75 & 86099.13 & $1.41 \mathrm{E}-11$ & 25464.19 & $2.36 \mathrm{E}-11$ \\
\hline 23.75 & 74252.27 & $1.48 \mathrm{E}-11$ & 24160.28 & $2.46 \mathrm{E}-11$ \\
\hline 26.75 & 64921.46 & $1.50 \mathrm{E}-11$ & 22349.71 & $2.68 \mathrm{E}-11$ \\
\hline
\end{tabular}




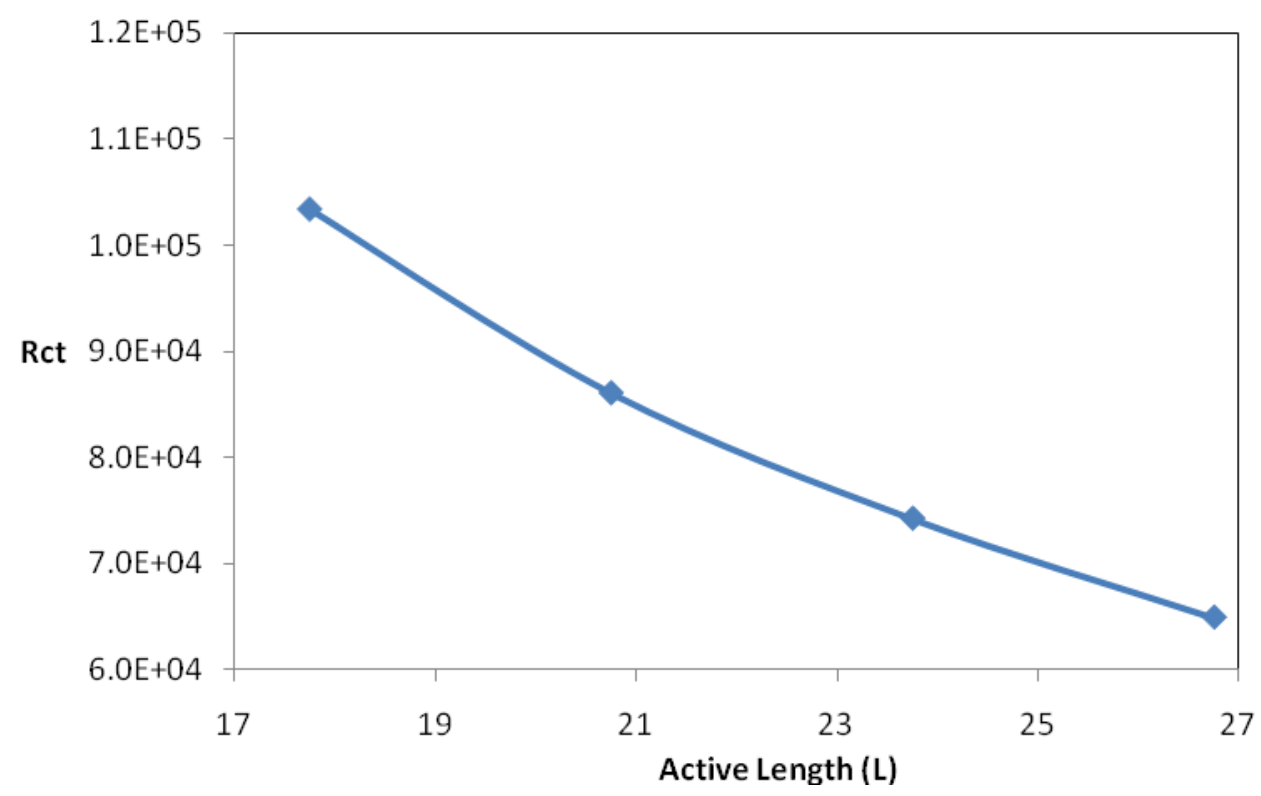

Figure 12. Charge Transfer Resistance variation with active length of Copper in DI water

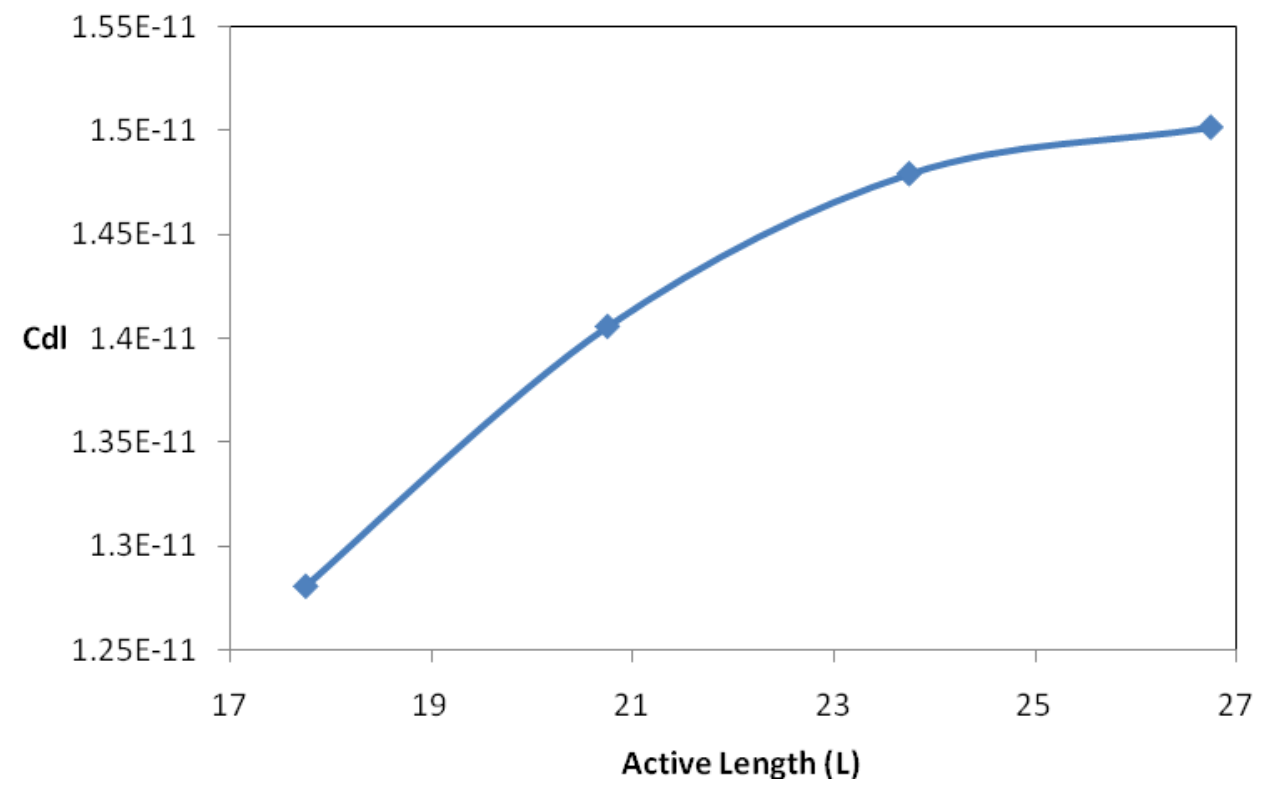

Figure 13. Double Layer Capacitance variation with active length of Copper in DI water

Diffusion phenomena were observed in section 3.1.2, where deionized water was used as electrolyte, while for section 3.2 (saturated $\mathrm{KCl}$ as electrolyte) there was no local diffusion effect.

Table 1 shows impedance parameters for the experiments, where DI water was used as the electrolyte, calculated by using equation (1) and (2). Our calculated Charge Transfer Resistance values are more or less equal to the diameter of respective semicircles of figure 5 and 6 , which means that, Randles circuit works well for the DI water case. We have roughly fixed Charge Transfer Resistance 
$\left(R_{c t}\right)$ and Double Layer Capacitance $\left(C_{d l}\right)$ values for each setup. But for experiments, where the $\mathrm{KCl}$ solution used as the electrolyte, both $R_{c t}$ and $C_{d l}$ varies with frequency and increases with a decrease of frequency, due to the formation of thin charge layers, as discussed earlier.

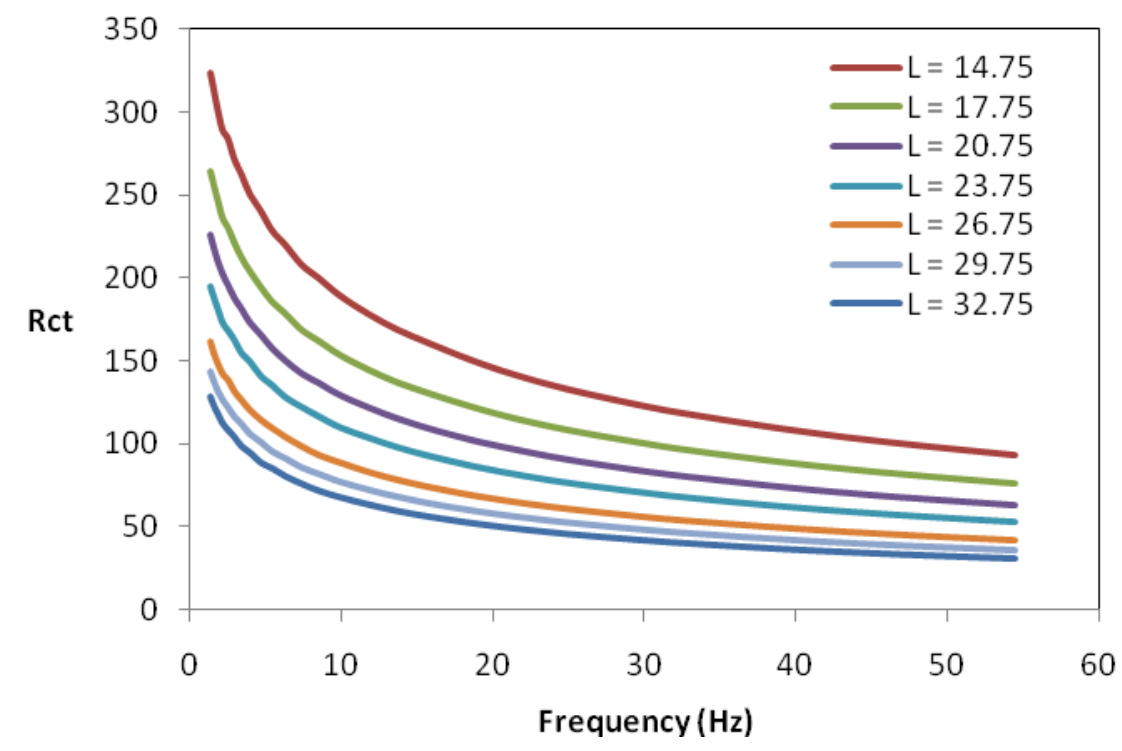

Figure 15. Charge Transfer Resistance variation for a range of frequency for various active length of copper.

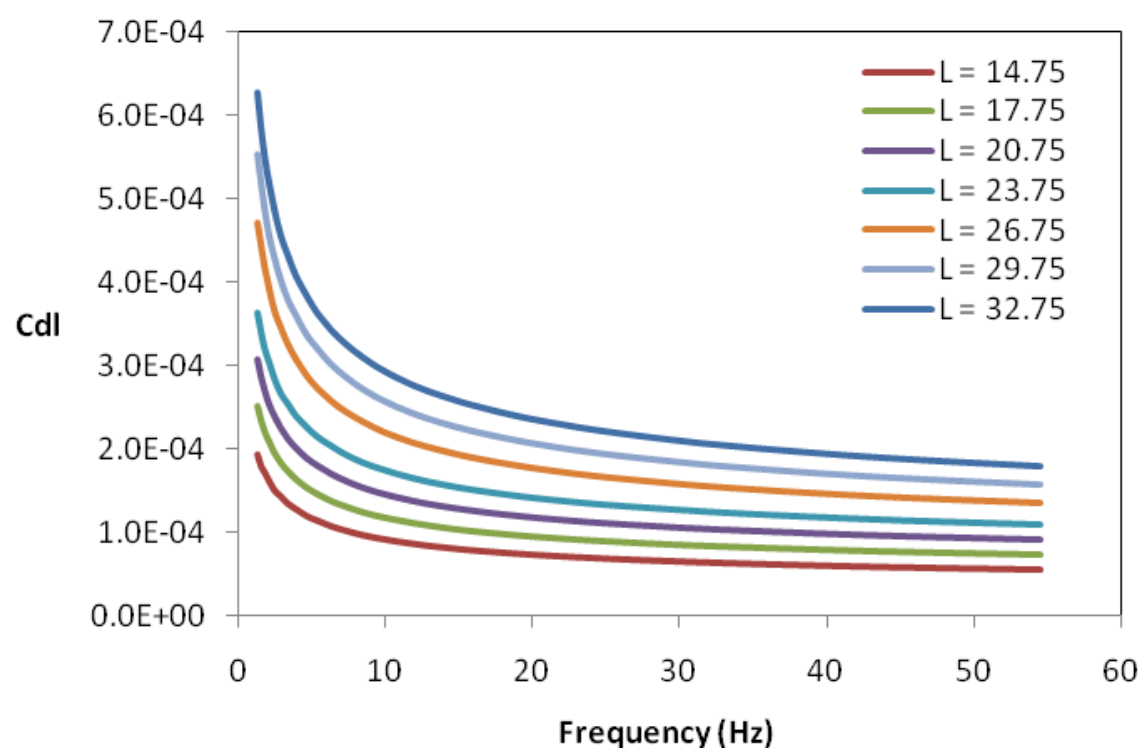

Figure 16. Double Layer Capacitance variation for a range of frequency for various active length of copper.

Figures 15 and 16 show changes of $R_{c t}$ and $C_{d l}$ for a small range of frequency. From Figures 15 and 16 it is also observed that charge transfer resistance shows decreasing values with increased active 
electrode area, as discussed. Conversely, the double layer capacitance increased with active area, reflecting the formation of local charge layers with increasing area. This behavior was also true for the DI water study, as shown in Figures 12 and 13.

\section{CONCLUSION}

Results obtained by systematically varying the geometric factors in an electrolytic cell have been identified. Some of those results are new, and others are consistent with published observations. As shown by V. Brichzin et al.[5], impedance in this situation is generally inversely proportional to active surface area, which we observed in our results. In the high frequency region, impedance parameters were more or less insensitive to active electrode area, but highly sensitive in the low frequency region. From our controlled results, it is evident that our experimental system works well and shows pure capacitive behavior in the case of alumina working electrodes. It also demonstrated Warburg diffusion in the case of DI water. For brass and copper electrodes, little or no local diffusion was observed for saturated $\mathrm{KCl}$ solution electrolyte. Using the Randell's equivalent circuit, the charge transfer resistance and double layer capacitance were found to be functions of frequency for the KCL electrolyte, , whereas for DI water these parameters were found to be roughly a fixed value. Both charge transfer resistance and double layer capacitance were found to follow a polynomial function with the alteration of active area, even for a purely capacitive system. From the literature, it is evident that charge transfer resistance shouldn't be a function a frequency. So it is obvious that the Randles circuit model doesn't work properly for the $\mathrm{KCl}$ solution case. We tried other models to fit the results and found none that work properly for the physical variables in our experiments. Of course, Randles circuit is a global approximation of system response, and is not likely to capture all of the local details under study here.

However, this work provides a foundation for understanding geometry effects on impedance response in the presence of active and passive local surfaces and with conductors and non-conductors. If a fundamental local understanding of the EIS data can be established, the present experimental method can be used not only to determine the basic nature of electrodes in working cells, but also as a foundation for the rational design and synthesis of electrodes with improved or specific performance. This foundation can also be used in future work in an effort to understand more complex geometry effects associated with local morphology of electrode materials for fuel cells and other functional material systems.

\section{NOMENCLATURE}

$R_{S} \quad$ Solution Resistance $(\Omega)$

$R_{c t} \quad$ Charge Transfer Resistance $(\Omega)$

$C_{d l} \quad$ Double Layer Capacitance (F)

$Z \quad$ Impedance $(\Omega)$

$Z_{r e} \quad$ Real Part of Impedance, Resistance $(\Omega)$

$Z_{i m} \quad$ Imaginary Part of Impedance, Reactance $(\Omega)$ 
$\varepsilon_{r} \quad$ Relative Static Permittivity

$\varepsilon_{0} \quad$ Electric Constant $(\mathrm{F} / \mathrm{mm})$

A Active Area of Electrode into Electrolyte $\left(\mathrm{mm}^{2}\right)$

$d \quad$ Distance Between Electrodes ( $\mathrm{mm}$ )

$L \quad$ Active Length of Electrode into Electrolyte (mm)

$M \quad$ Ion Travelling Distance (mm)

$D \quad$ Diameter of Electrode (mm)

\section{ACKNOWLEDGMENTS}

This work was supported by the grant from Department of Energy (DOE). USA

\section{References}

1. Rainer Küngas, Indrek Kivi and Enn Lust, J of ECS, Vol. 156 (2009) 345.

2. J. Winkler, P. V. Hendriksen, N. Bonanos, and M. Mogensen, J of ECS, 145 (1998) 1184.

3. S. H. Chan, X. J. Chen, and K. A. Khor, J. Appl. Electrochem., 31 (2001) 1163.

4. Cable, T.L., and Sofie, S.W., Journal of Power Sources, 174 (2007) 221.

5. V. Brichzin et al., Solid State Ionics, 152-153 (2002) 499.

6. B. W. Chung, A.-Q. Pham, J. J. Haslam, and R. S. Glass, J of ECS, 149 (2002) A325.

7. G. J. Offer, D. J. L. Brett, and N. P. Brandon, ECS Trans., 7(1), (2007) 1645.

8. G. Nurk, R. Küngas, I. Kivi, H. Kurig, V. Grozovski, S. Kallip, and E. Lust, ECS Trans., 7(1), (2007) 1609.

9. N. G. Minh and T. Takahashi, Science and Technology of Ceramic Fuel Cells, Elsevier, (1995).

10. M.E. Orazem and B. Tribollet, Electrochemical Impedance Spectroscopy, Wiley \& sons publication, (2008).

11. S. P. S. Badwal \& K. Foger, Ceramics International 22, (1996) 257.

12. Zhu and Kee, "Modeling Distributed Charge-Transfer Processes in SOFC Membrane Electrode Assemblies", J of ECS, 155(7), (2008) 715.

13. F. Zhao and A. Virkar, "Effect of Morphology and Space Charge on Conduction through Porous Doped Ceria," J. Power Sources, submitted Feb. (2010).

14. J. Maier, "Physical Chemistry of Ionic Materials," John Wiley \& Sons Ltd., England, (2004).

(C) 2011 by ESG (www.electrochemsci.org) 